\title{
Raw Data from HIPPARCOS Double and Multiple Stars
}

\author{
P. L. BERNACCA ${ }^{1}$, F. DONATI ${ }^{2}, \&$ F. MIGNARD ${ }^{3}$
}

${ }^{1}$ Asiago Astrophysical Observatory, University of Padova, 36012, Asiago, Italy

${ }^{2}$ Centro Studi sui Sistemi, via Vela 28, 20128, Torino, Italy

3 Observatoire de la Côte d'Azur, Department CERGA, 06130 Grasse, France

\begin{abstract}
The HIPPARCOS Input Catalogue comprises about 118,000 entries of which 16,000 are flagged as related to double or multiple stars. Data analysis begins by fitting Fourier model signals to the observations: the intensity coefficients and the phases at several locations on the spatial modulator are estimated and a first rough recognition of possibly unknown non-single stars is performed. After calibration corrections, a normal point is produced at every transit of a star across the field of view of the telescope and the improved parameters are stored for extensive multiplicity testing and relative astrometry when enough scans are accumulated.
\end{abstract}

\section{OBSERVATIONS OF DOUBLE AND MULTIPLE STARS}

The HIPPARCOS Input Catalogue (IC) is described in Perryman \& Turon (1989). In the course of the mission, which started in December 1989, it underwent some adjustments and debugging. The final version of the Catalogue has been published by ESA and the machine readable version can be retrieved from the Centre des Donnees Stellaire, Strasbourg. It comprises 118,260 entries among which 12,550 refer to double stars and 3,370 to multiple objects (see Table 1).

TABLE 1. Double and multiple star entries in the IC

\begin{tabular}{|cccr|}
\hline Type of entry & $\mathrm{N}$ & Type of entry & $\mathrm{N}$ \\
\hline $\mathrm{AB}$ & 7830 & $\mathrm{X}$ & 1760 \\
$\mathrm{~A}, \mathrm{~B}$ & 1120,1120 & $\mathrm{XY}$ & 1305 \\
$\mathrm{~A}$ & 2460 & $\mathrm{XYZ}$ & 287 \\
$\mathrm{~B}$ & 20 & $\mathrm{XYZW}$ & 18 \\
\hline$*$ & refer to multiple stars (e.g., $\mathrm{X}=\mathrm{A}$ or $\mathrm{B}, \mathrm{Y}=\mathrm{B}$ or $\mathrm{C}$, etc. \\
\hline
\end{tabular}

The actual number of distinct double stars is 11,430 and that of distinct multiples is 2,700. From Table 1 we see that for several double and multiple stars only one component is being observed.

Details on the HIPPARCOS telescope and on mission scientific operations can be read in Perryman \& Hassan (1989). It is sufficient to recall the following: the combined field of view (FOV) of the two viewing directions is $0.9 \times 0.9$ square 
degrees and a grid of 2,700 opaque and transparent bands is placed on the focal plane. The grid period is, nominally, $s=1^{\prime \prime} .208$. Because of the scanning motion of the satellite (at the nominal speed of $\omega=168^{\prime \prime} .75 / \mathrm{sec}$ ) the light from a star is modulated. Samples are collected with frequency $1,200 \mathrm{~Hz}$ and the detector window is switched to another star with frequency $150 \mathrm{~Hz}$. After 2.132 seconds (frame) the sequence repeats. Over a transit across the FOV, which lasts about $21 \mathrm{sec}$, there are, typically, 9 to 10 frames. In each frame 4 to 5 stars are observed.

The distribution of the separation between double and multiple star components and that of the magnitude difference is shown in Figure 1. Pairs of components separated by less than $10^{\prime \prime}$ are observed by letting the detector window (an Image Dissector Tube) follow the couple in its motion on the FOV according to the prescribed observing strategy. In the case of components in the separation range $10^{\prime \prime}$ to $30^{\prime \prime}$ (e.g., IC entries of type $(A, B)$ ), the two stars are observed alternatively, and for each of them the prescribed observing strategy is applied. When the separation is larger than $30^{\prime \prime}$, each component is observed like a single star.

\section{RAW DATA TREATMENT}

The modulated signal of a star is modeled by the following expected function (average count rate of a Poissonian distribution)

$$
S\left(t_{k}\right)=I+B+I M \cos \left(\omega t_{k}+\phi\right)+I N \cos 2\left(\omega t_{k}+\psi\right)
$$

where $t_{k}$ is the time at which the $k$-th sample is recorded, $\omega$ is the scanning velocity, $M$ and $N$ are modulation coefficients, $I$ is the intensity of the star $(\mathrm{Hz}), B$ is the background (nearly $40 \mathrm{~Hz}$ ) and $\phi$ and $\psi$ are phases. We have, typically, $I=1750 \mathrm{~Hz}$ for a star of $B=9$ and $B-V=0.6$.

When a star is not single, in relation (1) the intensity $I$ is the sum of the intensities af all components and the other quantities are related to those of the single components by the relations ( $i=1, . .$, number of the components)

$$
\begin{aligned}
I M \cos \phi & =\sum_{i} I_{i} M_{i} \cos \phi_{i} \\
I M \sin \phi & =\sum_{i} I_{i} M_{i} \sin \phi_{i} \\
I N \cos 2 \psi & =\sum_{i} I_{i} N_{i} \cos 2 \psi_{i} \\
I N \sin 2 \psi & =\sum_{i} I_{i} N_{i} \sin 2 \psi_{i}
\end{aligned}
$$

Examples of $S(t)$ for double stars are presented in Figure 2. At frame level (1 frame, as defined above, is the elementary observation made up of 2,560 samples) the following two estimation procedures are performed.

In the so-called 3-parameters model $S_{3}(k)$, the vector $\overrightarrow{b_{3}}(B, I, \phi)$ at frame mid-time is estimated, while $M, N$, and $v=\psi-\phi$ are retained as instrument 


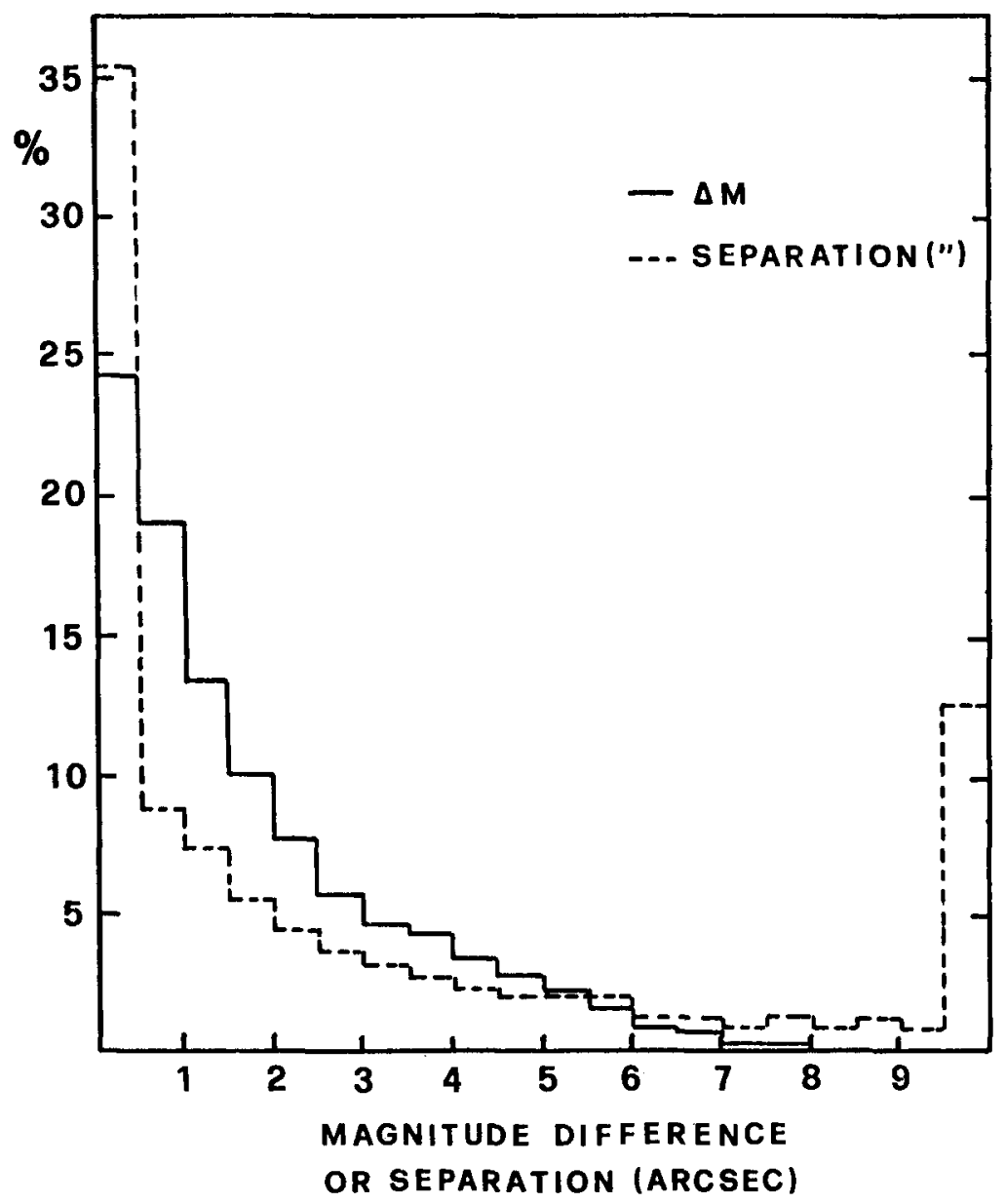

FIGURE 1. Distribution of the separation and magnitude difference between pairs of components in double and multiple star systems contained in the HIPPARCOS Input Catalogue.

constants which are duly calibrated on single stars along the mission and depend on field coordinates and star color. Here $v$ is a small quantity of order 1 mas due to diffraction chromaticity of the telescope and $M$ and $N$ are the telescope modulation coefficients (typically 0.72 and 0.28 respectively).

In the more general 5-parameters model $S_{5}(k)$, the vector $\overrightarrow{b_{5}}(I+B, I M, I N, \psi, \phi)$ is estimated. In both cases the estimation makes use of the maximum likelihood principle applied to a Poissonian distribution.

Implementation of the estimation requires a priori knowledge of star coordinates, satellite attitude and rate, field-to-grid transformation and grid calibration data. The actual procedure employes a modified expression (1), i.e. 
SINGLE STAR

$M=0.72 \quad N=0.26$

DOUBLE STAR

$M=0.50 \quad N=0.0$

$p=0.3$ arcsec

$\Delta M=0$
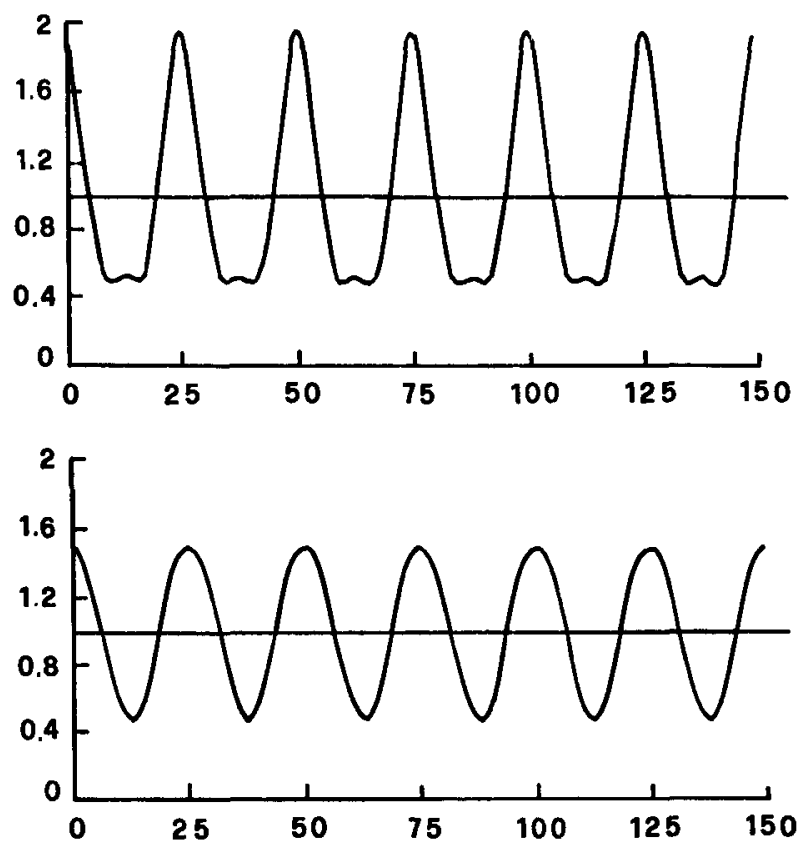

DOUBLE STAR

$M=0.36 \quad N=0.13$

$p=0.4$ arcsec

$$
\Delta \mathbf{M}=\mathbf{0}
$$

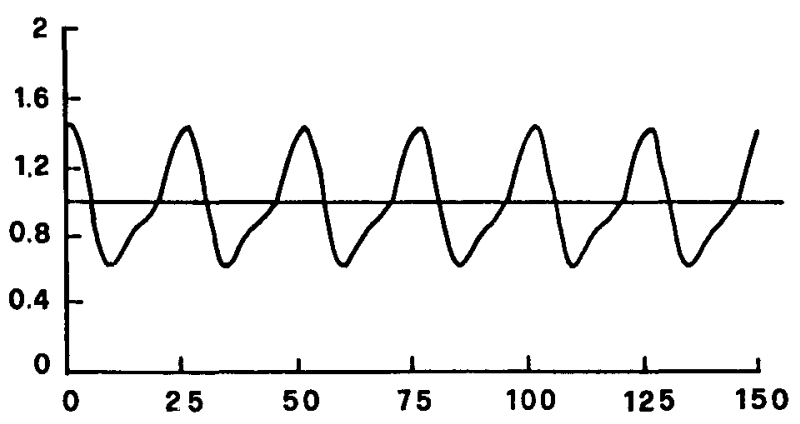

DOUBLE STAR

$M=0.18 \quad N=0.22$

$p=0.5$ arcsec

$\Delta M=0$

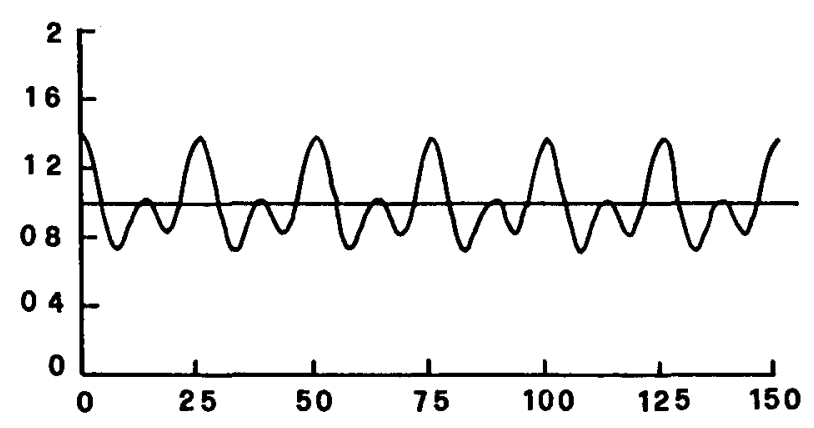

FIGURE 2. Examples of the signal expected function from double stars with different components separations $p$. In the case of the single star, $M$ and $N$ are the telescope modulation coefficient. 


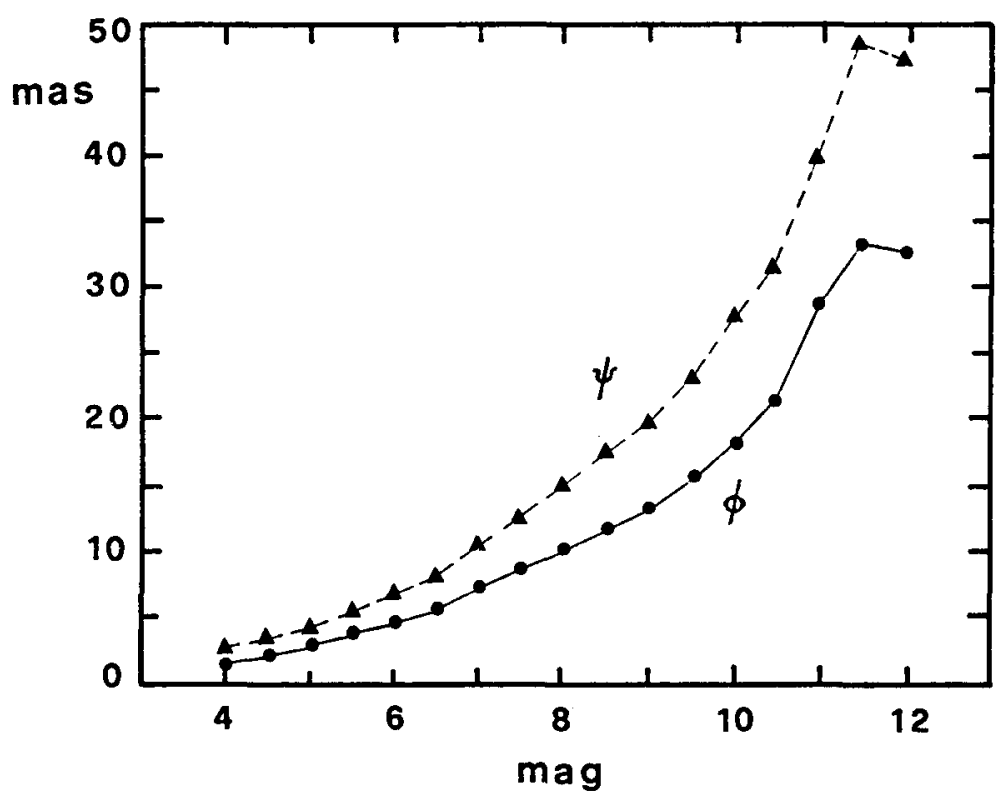

FIGURE 3. Error on the estimation of the phases and of the 5-parameter model of a star according to its magnitude (see text)

$$
S_{5}\left(t_{k}\right)=b_{1}+b_{2} \cos p_{k}+b_{3} \sin p_{k}+b_{4} \cos 2 p_{k}+b_{5} \sin 2 p_{k}
$$

where $p_{k}=2 \pi \omega t_{k} / s, s$ being the grid period , and the five parameters $b_{1}, \ldots, b_{5}$ being related simply to the previous components of $\overrightarrow{b_{p}}(\mathrm{p}=3,5)$. The estimation procedure is discussed in detail by Murray et al. (1989) and it is based on a least-squares solution followed by a Gauss-Markov approach. With real data the phases are beeing obtained with the precision shown in Figure 3.

Null hypotheses like "the signal is not modulated" and "the p-parameters model is true" are tested by suitably defined indices which obey pseudo-Gaussian statistics. Of particular relevance to non-single stars detection is the following F35 index

$$
F_{35}=\left[\frac{1}{2} \sum_{k=1}^{N} \frac{\left(S_{5}(k)-S_{3}(k)\right)^{2}}{S_{3}(k)}\right]\left[\frac{1}{N-5} \sum_{k=1}^{N} \frac{\left(N_{k}-S_{5}(k)\right)^{2}}{S_{5}(k)}\right]^{-1}
$$

where $\mathrm{N}$ is the total number of samples and $N_{k}$ are the observed counts/sample. Under the null hypothesis that $S_{3}(k)$ holds, $F_{35}$ follows a Fischer-Snedecor distribution with 2 and $N-5$ degrees of freedom. The null hypothesis is rejected when $F_{35}$ is larger than 7 which corresponds to a probability of 0.1 percent of making a wrong decision. The risk would raise to 0.5 percent with a treshold of 5. Among the stars flagged as single in the Input Catalogue, typically about 15 percent are found positive to this test, which means that the number of double or multiple stars in the IC might be much larger than reported in Section 1. It 
should, however, be remarked that rejection of the $S_{3}(k)$ model does not necessarily imply the presence of a non-single star. For, indeed, departure from a simple signal may also be due to spurious companion stars imaged by the other field of view or to "veiling glare" caused by bright objects in the wings of the detector's window.

\section{DATA CONDENSATION}

The vector $b_{5}$ is available per observation frame and there are 9 frames per transit (or 5 in the case of the alternate strategy). The quantities $\phi-\phi_{i}$ and $\psi-\phi_{i}$, hence $\psi-\phi$, depend only on the structure of the double (or multiple) star image and remain constant over short time intervals. Therefore, all frames in a transit are combined together in order to reduce statistical fluctuations and the amount of input data to subsequent processing. To this purpose the phase of the first harmonic is chosen as reference point internal to the double star and relations (2) are rewritten accordingly. In the right hand side, quantities like $\phi_{i}-\phi$ appear and they become the unknowns of the intermediate problem of knowing the projected separation along the scanning direction modulo the grid period, given simply by their difference. In addition the $\psi_{i}$ are replaced by $\Phi_{i}+v$ (and $v$ may eventually be neglected). The left hand side of relations (2) becomes $(r=1$, number of frames $n)$

$$
\begin{aligned}
(\widehat{I M})_{\boldsymbol{r}} \cos \left(\hat{\phi}_{\boldsymbol{r}}-\phi_{\boldsymbol{r}}\right) & =A \\
(\widehat{I M})_{\boldsymbol{r}} \sin \left(\hat{\phi}_{\boldsymbol{r}}-\phi_{\boldsymbol{r}}\right) & =B=0 \\
(\widehat{I N})_{\boldsymbol{r}} \cos 2\left(\hat{\psi}_{\mathbf{r}}-\phi_{\boldsymbol{r}}\right) & =C \\
(\widehat{I N})_{\boldsymbol{r}} \sin 2\left(\hat{\psi}_{\boldsymbol{r}}-\phi_{\boldsymbol{r}}\right) & =D
\end{aligned}
$$

where the symbol ' indicates values estimated at frame level. We have $3 n$ equations, $n$ constraints $(B=0)$ and $n+3$ unknowns (i.e. the phases $\phi_{r}$ and $A$, $C, D$. The solutions are found by minimizing the weighted $\chi^{2}$ through a firstorder fixed-point iteration and a second-order Newton-Rapson method. The errors on $A, B, C$, and $D$ are computed from the correlation matrix obtained by inverting the Jacobian.

Before performing the condensation, $(\widehat{I M})_{r},(\widehat{I N})_{r}$, and $\hat{\phi}_{r}$ are to be corrected for calibration of the instrument. In principle the correct value $(I M)_{c}$ at the FOV center would be given, in the case of a double star, by (we neglect the frame index $r$ )

$$
\begin{aligned}
(I M)_{c}^{2} & =I_{1}^{2} M_{1 c}^{2}+I_{2}^{2} M_{2 c}^{2} \\
& +M_{1 c} M_{2 c}\left[(\widehat{I M})^{2}-R_{1}^{2} F^{2}\left(d_{1}\right) I_{1}^{2} M_{1}^{2}-R_{2}^{2} F^{2}\left(d_{2}\right) I_{2}^{2} M_{2}^{2}\right] \\
& \times\left[R_{1} R_{2} F\left(d_{1}\right) F\left(d_{2}\right) M_{1} M_{2}\right]^{-1}
\end{aligned}
$$

where the quantities not previously defined have the following meaning:

$\star M_{1 c}$ and $M_{2 c}$ are the telescope modulation coefficients at the FOV center for the first and second component. Calibration tables allow to compute $M_{1}$ and $M_{2}$ everywhere in the FOV as a function of the star color $B-V$. 
$\star R$ is a function which relates the intensity $I$ at the FOV center to that everywhere on the FOV and it is color dependent.

$\star F(d)$ is the efficiency profile of the Image Dissector Tube, $d$ being the distance of a star image from the center of the $38^{\prime \prime} \times 38^{\prime \prime}$ detection window.

The corrective terms in relation (6) depend strongly on the a priori knowledge of the magnitudes of each component which are needed to compute the I's and these are not sufficiently precise. In addition, due to the small separations of the components, $R_{1}-R_{2}$ and $M_{1}-M_{2}$ will differ from zero mainly because of the color dependence. It is $d R / d(B-V)=d M / d(B-V)=0.08 /$ magnitude. Since colors of double and multiple stars components are not generally available and because the great majority of double star components have similar colors, it has been decided to assume a unique function $R$ and a unique function $M$ and, in addition, to ignore $F(d)$, that is to take unity. Relation (6) becomes very simple and it is also used for multiple stars. The color of the primary is used to compute $R$ and $M$ values from the calibration files. The differences $\Delta R$ and $\Delta M$ reach about -20 percent of their central value near the FOV edges.

With this procedure the intensities $I-i$ that are derived by further processing relations (2) will be the intensities of the components scaled by the factor $F(d)$ which depends on the actual positioning of the detector's window which follows the star along the transit. As photometry is not an outcome of double or multiple star astrometrical processing, we may ignore this fact.

The same relation applies to the correction of $(I N)$. In addition to known double and multiple stars, all stars filed as single in the Input Catalogue are treated with the simplified relation (6), which holds strictly if a star is single, with a view to further testing of their possible duplicity.

As far as corrections to the estimated phases in the $S_{5}$ model are concerned, it turned out that the second harmonic of single star signals is systematically shifted by about 21 mas due to deformations of the optics after launch. It is given at any FOV position and the correction is decreasing with time. This effect is simply subtracted from $\hat{\psi}_{r}$ which is correct when the components have similar colors. In the case of different colors the uncertainty in the correction is only of a few mas and smaller than the random error shown in Figure 3.

The condensed parameters $A, B, C$, and $D$ and one of the improved phase $\phi_{r}$ are stored for furhther processing of the modified equations (2) when enough scans are accumulated (see Mignard et al. 1989). Improved values of $\phi-r$ are also obtained from $\phi_{r}$ by combining the two last equations (5) and one of the phases is also stored for use in the imaging approach to multiple star processing (see Mignard et al. 1989).

\section{REFERENCES}

Mignard, F., Borriello, L., Kovalevsky, J., Prezioso, N., \& Bernacca, P.L. 1989, in The HIPPARCOS Mission, ESA-SP 1111, 3, 243

Murray, C.A., van Leuwen, F., Lindegren, L., Donati, F., \& Fassino, B. 1989, in The HIPPARCOS Mission, ESA-SP 1111, 3, 33

Perryman, M.A.C. \& Hassan, H. (Eds.) 1989, The HIPPARCOS Mission, ESA-SP 1111,1 , "The HIPPARCOS Satellite"

Perryman, M.A.C. \& Turon, C. (Eds.) 1989, The HIPPARCOS Mission, ESA-SP 1111, 2, "The Input Catalogue" 